\title{
Maladie parodontale et syndrome de Takayasu
}

\section{RÉSUMÉ}

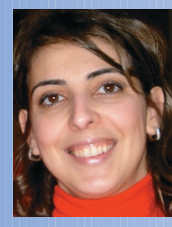

Zineb AZZIM AN

Spécialiste en parodontologie, Service de Parodontologie,

Centre de consultation et de traitement dentaire de Rabat,

CHU Ibn Sina - Rabat, Maroc.

9, rue Acaena Riad 3

Rabat, Maroc.

\section{Amine CHERKAOUI}

Professeur agrégé de parodontologie, Service de parodontologie,

Centre de consultation et de traitement dentaire de Rabat,

CHU Ibn Sina - Rabat, Maroc.

\section{Latifa BENRACHADI}

Professeur de l'enseignement supérieur, Service de parodontologie,

Centre de consultation et de traitement dentaire de Rabat,

CHU Ibn Sina - Rabat, Maroc.

\section{Oum Keltoum ENNIBI}

Professeur de l'enseignement supérieur et chef de service de parodontologie

de Rabat,

Service de parodontologie,

Centre de consultation et de traitement dentaire de Rabat,

CHU Ibn Sina - Rabat, Maroc.

Les parodontites sont des infections multifactorielles des tissus de soutien des dents. Les biofilms bactériens jouent un rôle essentiel dans le déclenchement et la propagation de ces infections. Plusieurs pathologies systémiques peuvent également influencer ces parodontites ou encore être aggravées par ces dernières.

Nous nous intéressons dans cet article à une maladie rare, l'artérite de Takayasu. Il s'agit d'une vascularite inflammatoire et sténosante des artères de moyen et de gros calibres qui se manifeste par différents signes cliniques locaux et généraux.

À travers une observation clinique approfondie d'un patient rencontré au CCTD de Rabat, nous avons essayé d'éclaircir une éventuelle relation entre le syndrome de Takayasu et les infections parodontales, ainsi que les particularités de la prise en charge parodontale chez ces patients, qui doit prendre en considération les différentes contraintes liées, d'une part, à la maladie et ses différentes manifestations, et d'autre part, aux traitements administrés à ces malades.

- parodontite

- artérite de Takayasu

prise en charge 


\section{Introduction}

$>$

Les parodontites sont des infections multifactorielles des tissus de soutien des dents. Quelques bactéries sous-gingivales du biofilm dentaire jouent un rôle essentiel dans le déclenchement et la progression de la maladie. D'autres facteurs, tels que le tabac, le stress, certaines maladies systémiques, des dysfonctionnements du système immunitaire de I'hôte, ainsi que des facteurs génétiques peuvent profondément influencer le cours de cette maladie[1, 2].

Au cours de la dernière décennie, de nombreuses études ont corrélé les parodontites aux pathologies systémiques, ce qui a donné naissance à une nouvelle branche de la parodontologie: la médecine parodontale[3]. Cette notion introduite par Offenbacher, et coll. en 1996 correspond à une double relation dans laquelle les maladies parodontales ont une influence nocive sur certaines maladies systémiques et les pathologies générales peuvent aggraver, par certains mécanismes, l'évolution des parodontites[4].

L'intérêt a été porté dans cet article sur une pathologie rare qui est le syndrome de Takayasu. C'est une pathologie idiopathique découverte en 1908 par l'ophtalmologiste japonais Takayasu. Elle se définit comme une vascularité inflammatoire et sténosante des artères de moyen et de gros calibre, caractérisée par une atteinte préférentielle de la crosse de l'aorte et de ses branches[5].

À travers une observation clinique, nous allons tenter d'éclaircir deux points :

- la relation entre la maladie de Takayasu et la maladie parodontale ;

- les particularités de la prise en charge parodontale chez les patients atteints de cette maladie.

\section{Syndrome de Takayasu}

L'artérite de Takayasu est une maladie systémique associant des signes généraux et des symptômes en rapport avec une atteinte locale (fig. 1).

Les signes généraux allient malaise, fièvre, sueurs nocturnes, arthralgies, anorexie et amaigrissement, qui peuvent apparaître plusieurs mois avant que l'atteinte vasculaire ne soit évidente.

Les symptômes locaux sont ceux d'une atteinte vasculaire et débutent par des douleurs, suivies par des signes d'ischémie des organes vascularisés par les vaisseaux atteints.

Les pouls sont habituellement abolis, en particulier celui de l'artère sous-clavière. Une insuf- fisance aortique peut apparaître et l'hypertension artérielle est observée chez environ $50 \%$ des patients[6].

L'atteinte des artères carotides est responsable de différents tableaux cliniques. Environ la moitié des patients présenteront des syncopes; on observe également des accidents vasculaires cérébraux chez $15 \%$ des patients; les manifestations oculaires quant à elles, sont présentes dans $60 \%$ des cas.

Les examens biologiques mettent en évidence une accélération de la vitesse de sédimentation, une anémie modérée et un taux élevé d'immunoglobuline. 


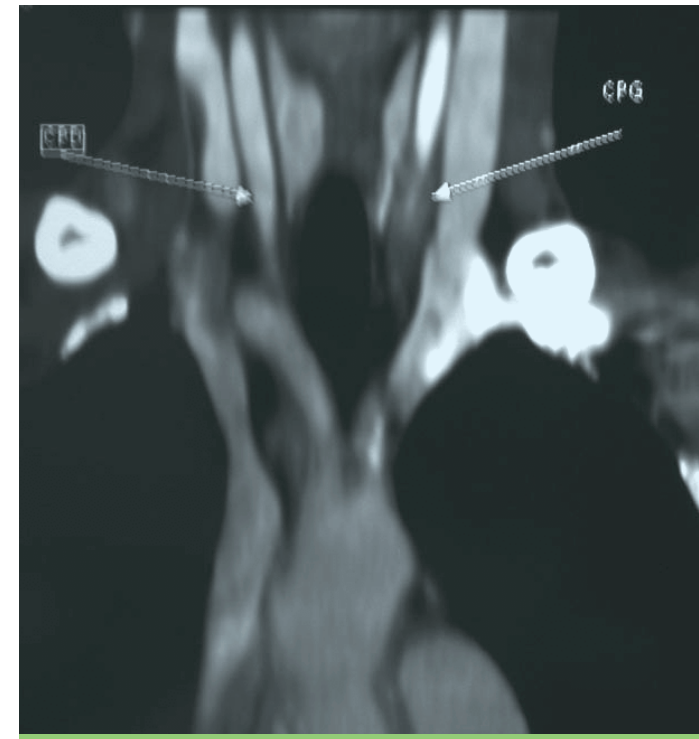

Fig. 1 Angio-scanner des vaisseaux supra-aortiques affirmant le diagnostic de sténoocclusion des carotides primitives notamment dans leurs portions cervicales.

\section{Observation clinique}

Un patient âgé de 41 ans s'est présenté au centre de consultations et de traitements dentaires de Rabat-Salé.

Lors de l'entretien clinique, le patient rapporte la présence d'une artérite de Takayasu révélée par des pertes de connaissances syncopales et traitée par une corticothérapie au long cours à la dose de 40 mg de prédnisone par jour, associée à un traitement antiagrégant plaquettaire à base d'acide acétyl salicylique à la dose de $250 \mathrm{mg}$ par jour. Le patient est non-fumeur.

L'examen exobuccal ne présente aucune particularité.

À l'examen parodontal, l'indice de plaque et l'indice gingival de Loe et Silness sont assez élevés[2,5], avec présence de dépôts tartriques généralisés (fig. 2).

L'examen de la gencive montre une inflammation généralisée avec présence d'œdèmes
L'évolution de la maladie peut être fulminante, progressive ou stable. Les complications dépendent directement de la localisation de l'atteinte vasculaire. L'insuffisance cardiaque congestive ou les accidents vasculaires cérébraux constituent les causes habituelles de décès.

Le traitement de la maladie de Takayasu est basé sur l'association de la corticothérapie au long cours à la dose de 40 à 60 mg de prédnisone liée à un traitement chirurgical et/ou angioplastique des sténoses vasculaires. Chez les patients corticorésistants, les immunosuppresseurs (le méthotrexate) à la dose de $25 \mathrm{mg} / \mathrm{semaine}$ donnent des résultats encourageants. Un traitement antiagrégant plaquettaire est également associé[5].

papillaires et de saignements au sondage. La pression gingivale fait sourdre un exsudat purulent important.

Le sondage parodontal met en évidence des pertes d'attaches généralisées avec présence de poches parodontales variant entre 5 et $10 \mathrm{~mm}$ au maxillaire supérieur. Les récessions

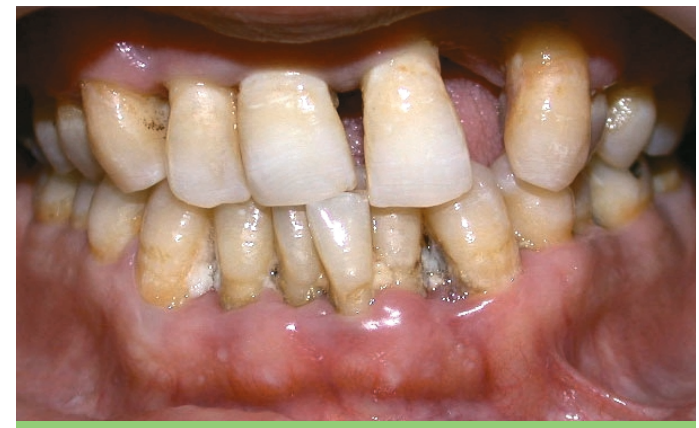

Fig. 2 Vue endobuccale montrant la présence des dépôts locaux associés à une inflammation gingivale généralisée. 


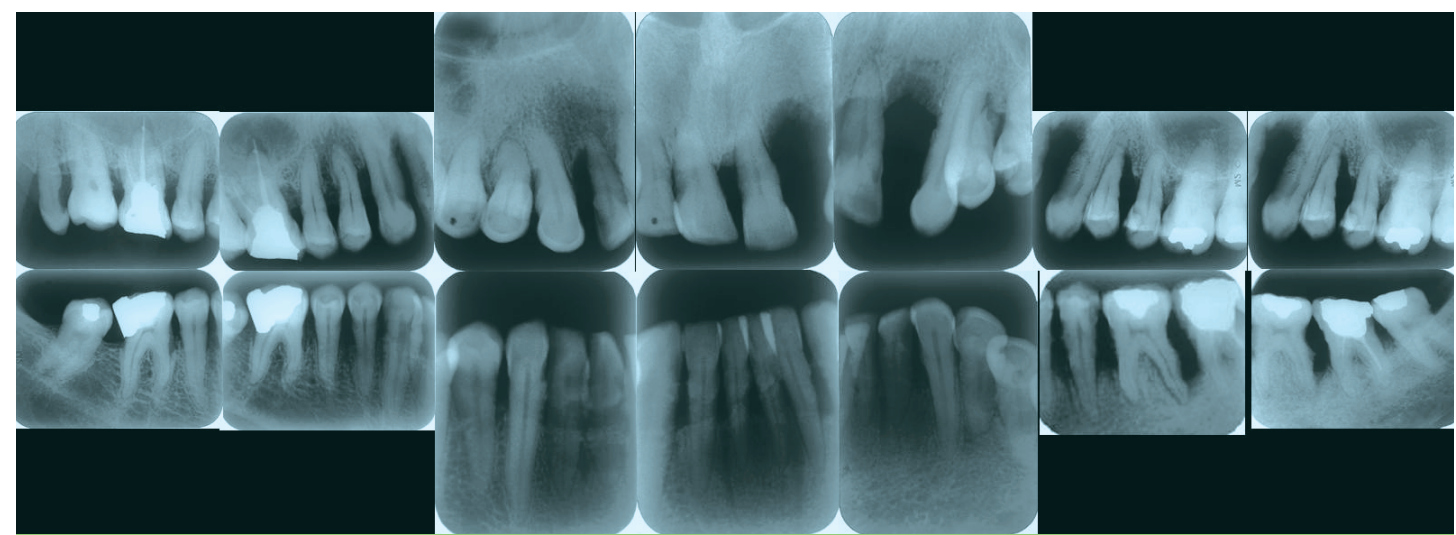

Fig. 3 Bilan rétro-alvéolaire long cône montrant des lyses osseuses généralisées modérées à sévères.

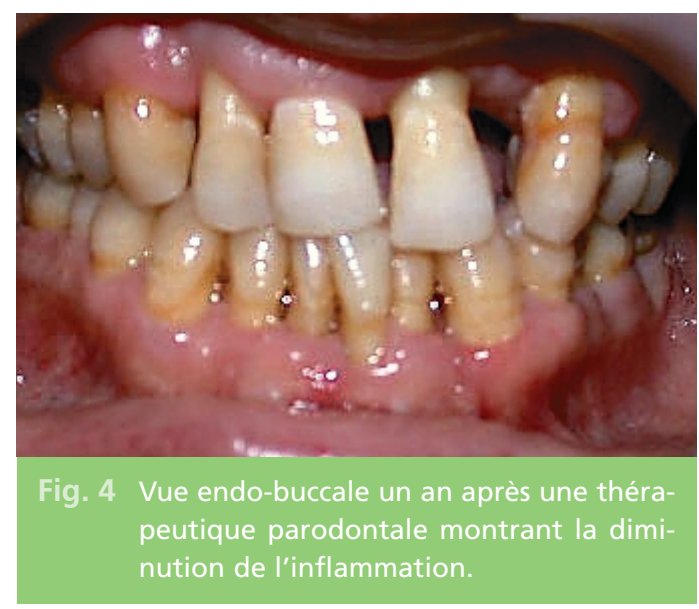

parodontales de classe III de Miller existent sur la grande majorité des dents mandibulaires sans présence de poches parodontales.

Sur le plan muco-gingival, la gencive est de hauteur suffisante sauf au niveau des incisives mandibulaires.

L'évaluation de la mobilité dentaire révèle la présence de mobilités de degré 2 de Nyman au niveau des incisives et des canines supérieures et inférieures.

L'examen radiologique à base d'un bilan rétroalvéolaire au long cône montre une lyse osseuse horizontale généralisée à mi-hauteur radiculaire, avec des foyers verticaux et de lyses terminales ponctuelles au niveau de la 12, 24 et 36 (fig. 3).

Vu ce qui précède, et la forte corrélation entre les dépôts et la sévérité des lésions, le diagnostic d'une parodontite chronique modérée à sévère généralisée a été retenu.

Un traitement parodontal a été réalisé chez ce patient, dans le but de rétablir la santé parodontale, diminuer l'inflammation gingivale et améliorer les conditions cliniques.

La motivation et l'enseignement aux techniques d'hygiène orale, ainsi que l'élimination des facteurs de rétention de la plaque par un détartrage et des soins de caries ont fait l'objet de la première étape du traitement. Le sur- 
façage radiculaire a fait suite à cette étape en sachant que tous ces soins ont été réalisés sous antibioprophylaxie (Amoxicilline $2 \mathrm{~g}$ par jour en deux prises, pendant une semaine, débutée deux jours avant l'intervention) et après arrêt des anticoagulants ( 10 jours avant chaque séance) suivant les recommandations de son médecin traitant.

\section{Discussion}

La maladie de Takayasu est une panartérite caractérisée par une infiltration et une prolifération des cellules mononucléées inflammatoires et occasionnellement par des cellules géantes aboutissant à une fibrose de l'intima, des lésions séquellaires de la média, une fragmentation et une dégénérescence de la limitante élastique[7].

La lumière du vaisseau ainsi réduite sera associée ultérieurement à une thrombose.

L'atteinte des vasa vasorum (artérioles permettant la nutrition de la paroi des gros vaisseaux) est fréquente par un infiltrat de cellules pluripotentes (lymphocytes T, granulocytes et monocytes) avec production de cytokines générant ainsi une réponse inflammatoire et entraînant les syndromes "vascularitiques» décrits dans cette pathologie.

Les causes de la maladie de Takayasu restent encore obscures, et les mécanismes pathogéniques corrélant cette artérite et la maladie parodontale ne sont pas clairement définis. Mais la réaction auto-immune à certaines infections a été évoquée, comme la maladie parodontale qui reste une infection polymicrobienne chronique. En effet, Franklin et Morrisson[8] ont démontré l'existence d'une réaction entre les lymphocytes du malade et
Bien qu'une certaine amélioration des paramètres cliniques ait été obtenue chez ce patient, les résultats de la thérapeutique parodontale étaient loin d'être optimaux, ce dernier a donc été réorienté vers une thérapeutique parodontale de soutien palliative (fig. 4).

les antigènes bactériens et ont remarqué que les protéines inflammatoires produites ont participé à la propagation de la maladie de Takayasu par destruction des fibres élastiques péri-vasculaires.

Dans l'autre sens, le syndrome de Takayasu peut à son tour avoir un impact sur la maladie parodontale d'autant plus que la vascularisation nourricière du parodonte provenant de la carotide externe et de ses ramifications est généralement atteinte dans le type III de la maladie de Takayasu.

Aussi, les traitements administrés chez ces malades sont essentiellement basés sur les corticoïdes et les immunosuppresseurs et la relation entre la prise médicamenteuse et la maladie parodontale a été largement documentée[9]. En effet, ces traitements ont pour effets secondaires d'augmenter le risque infectieux et de diminuer les défenses naturelles de l'organisme. Ils agissent en réduisant ou en supprimant les réactions immunologiques spécifiques de l'organisme contre un antigène par blocage des systèmes de défense immunitaires humoral et cellulaire, ce qui peut influencer le développement et la progression de la maladie parodontale ainsi que les résultats des traitements[10]. 
Enfin, la chronicité de la maladie et l'observance d'un traitement pendant des années, peuvent être à l'origine d'anxiété et d'angoisse générant un désintérêt pour le suivi parodontal, d'où l'intérêt d'une prise en charge pluridisciplinaire par une équipe opératoire, associée à un soutien psychologique le cas échéant.

Il en résulte donc que la prise en charge parodontale des patients atteints de la maladie de Takayasu doit prendre en considération les données sus-citées, le contexte psychologique, ainsi que les traitements en cours afin d'éviter tout risque infectieux, hémorragique ou syncopal, ce qui a donné lieu aux règles suivantes[11, 12] :

- en premier lieu, les soins parodontaux doivent être réalisés en dehors des phases aiguës de la maladie ;

\section{Conclusion}

L'association maladie parodontale et artérite de Takayasu a été très rarement décrite. Cette entité s'inscrit dans le cadre de l'association maladie parodontale et pathologies vasculaires inflammatoires.

L'intérêt de cette observation clinique réside dans la vulgarisation de cette pathologie, certes rare, mais qui reste assez spécifique dans
- les patients présentant une hyperpression du sinus carotidien (souvent atteint dans la maladie de Takayasu) doivent être installés dans la position la plus adéquate qui permet un relâchement de la région du sinus carotidien ;

- en raison des pouls diminués ou absents, un monitoring est nécessaire en peropératoire afin de permettre une surveillance plus concrète du patient ;

- une antibioprophylaxie reste de mise en raison de la prise d'anti-inflammatoires stéroïdiens pendant une longue durée chez ces patients, afin de prévenir tout risque infectieux ;

- enfin, l'arrêt des anticoagulants contrôlé par des examens de la crase sanguine répétés est nécessaire pour prévenir le risque hémorragique chez ces patients.

ses traitements dentaires et parodontaux. Ces derniers doivent faire appel à une équipe médicale pluridisciplinaire travaillant de concert afin d'optimiser la prise en charge de ces patients. Enfin, il faut insister sur l'importance d'un soutien moral continu en raison du contexte physique et psychologique affaibli que présentent ces patients.

\section{Bibliographie}

1. Van Winkelhoff AJ, Van Der Reijden WA. Infections parodontales et implications thérapeutiques. J Parodontol Imp Orale 2000;19(2).
2. Baetigny $\mathrm{H}$, Delcourt-Debruyne E. Étiologie des parodontites. Facteurs généraux 
et locaux de susceptibilité aux parodontites.

Encycl Méd Chir

(Elsevier, Paris) 23-435-A-10.

3. Micheau C, Ouhayoun JP. Influences des parodontites sur les pathologies systémiques.

J Parodontol Imp Orale 2001;20(4):293-303.

4. Offenbacher $\mathrm{S}$, et al.

Periodontal diseases:

pathogenesis.

World workshop

in periodontics.

Ann Periodontol 1996;1:

821-878.

5. Quemeneur T, Hachulla E, Lambert M, Perez-Cousin M, Queyrel V, et al.

Takayasu arteritis.

Press Med 2006;35:847-856.

6. Lin Jian, Chen Bin, Wang Jian-Hua.
Diagnosis of systemic

arterial diseases

with whole-body 3D

contrast-enhanced

magnetic resonance

angiography.

Chinese medical journal:

12/2006;119(21):172-8.

7. Kamaoui l, et al. Ischemic stroke caused by Takayasu's arteritis. J Mal Vasc 2007;32(1):43-46.

8. Franklin et Morrisson. Surgical management of Takayasu's arteritis. J Korean Med Sci 2006;21:20-24.

9. Hassell T, et al. Facteurs de risque parodontaux associés aux traitements médicamenteux chez les patients âgés.
Parodontol Implant Orale 1998;17(2).

10. Le Toux G, Bonnaure-Mallet $\mathrm{M}$, Abjean J. Hyperplasie gingivale sous Ciclosporine A.

Données actuelles et rapport d'un cas. J Parodontol 1990;9(2): 155-159.

11. Yoshimura $Y$, et al. Tooth extractions and aortitis syndrome (Takayasu's disease). Int J Oral Surg 1981;10(1): 1-11.

12. Duque $C$, Silva $R C$, Santos-Pinto L. Takayasu's arteritis: what should the dentist know? Int J Paediatr Dent 2005;15(2):113-117.

\section{SUMMARY}

\section{Periodontal disease and Takayasu arteritis}

Zineb AZZIMAN, Amine CHERKAOUI, Latifa BENRACHADI,

Oum Keltoum ENNIBI

$$
\begin{aligned}
& \text { Keywords } \\
& \text { - periodontitis } \\
& \text { - Takayasu arteritis } \\
& \text { - managing } \\
& \text { and treatment }
\end{aligned}
$$

Periodontitis are multifactorial infections of teeth supporting tissues. The bacterial biofilms have an essential role in the begining and propagation of these kind of infections.

Several systemic diseases can also influence these periodontitis and worsen the outcome.

The aim of this article is the study of a rare disease: the Takayasu arteritis (wich is a sténosing inflammatory vascular disease of midle and large arterities).

We report a case of a patient treated in the center of dental diseases at Rabat (Morrocco).

we tried to clear up the possible relationships between the Takayasu arteritis and periodontal infections, and highlight on the treatement according to the particularities of both of the two entities. 


\section{Questions - Réponses}

1/ Réponse par vrai ou faux

a - La maladie de Takayasu est une pathologie idiopathique
Vrai
Faux

b - La maladie de Takayasu est une vascularite inflammatoire et sténosante des artères de petit calibre

Vrai

Faux

c - L'hypertension artérielle est observée chez tous les patients

Vrai $\square \quad$ Faux

2/ QCM à une seule réponse

A - Les signes généraux du syndrome de Takayasu sont :

a - fièvre avec sueurs nocturnes

b - des arthrites mécaniques

c - une hypotension orthostatique

d - une prise de poids chez le patient

B - Les soins parodontaux chez les patients atteints du syndrome de Takayasu :

a - sont strictement contre-indiqués

b - nécessitent une antibioprophylaxie

c - sont toujours réalisés en milieu hospitalier

d - l'arrêt des anti-coagulant n'est pas obligatoire 